\title{
Proyecto de Reformas al Protocolo de Brasilia. Una nueva oportunidad perdida
}

Alejandro Daniel Perotit *

SUMÁRIO: 1. Introdução. 2. O Projeto de Reformas ao Protocolo de Brasilia. 3. O Projeto de Reformas e a Decisão 25/00 CMC. 4. Consideraçóes Globais sobre o Projeto de Reformas. 5. Anexo: Sobre o Mito da não-necessidade do Tribunal de Justiça do MERCOSUL 6. Conclusão. 7. Bibliografia.

SUMMARY: 1. Introduction. 2. The Project of Reformations to the Protocol of Brasilia (PRPB). 3. The Project of Reformations and the Decision 25/00 CMC. 4. Global Considerations about the Project of Reformations. 5. Annex: On the Myth of the innecesariedad of court of justice in MERCOSUR. 6. Conclusion. 7. Bibliography.

SUMARIO: 1. Introducción. 2. El Proyeto de Reformas al Protocolo de Brasília (PRPB). 3, El Proyeto de Reformas y la decisión 25/00 CMC. 4. Consideraciones Globales acerca del Proyeto de Reformas. 5. Anexo: Sobre el Mito de la innecesariedad de Tribunal Justicia del MERCOSUR 6. Conclusión. 7. Bibliografia.

RESUMO: Este artigo refere-se a polêmica discussão sobre o Protocolo de Brasília e o seu mecanismo de solução de controvérsia. Embora o protocolo de Brasillia tenha sido revogado pelo protocolo de Olivos, que aborda a mesma matéria, as questóes levantadas continuam sendo de alta importância para a necessidade permanente de aperfeiçoamento do sistema jurídico do MERCOSUL, que demanda a institucionalização de um tribunal de justiça a exemplo do europeu.

\footnotetext{
* Master en Derecho Comunitario de la Cniv. Complutense de Madrid, España Profesor de Defucho de la Integración de la Univ. Austral, Buevos Aires. Ematl adperotiogyhoocom.
} 
ABSTRACT: This article refers the polemic discussion on the protocol of Brasilia and its mechanism of solution of controversy. Although the protocol of Brasilia has been revoked by the protocol of Olivos, that approaches the same matter, the lifted up subjects continue being of high importance for the permanent need of improvement of the juridical system of MERCOSUR, that demands institutionalization of a tribunal of justice to example of the european or andine.

RESUMEN: Este artículo refiere la discusión polémica sobre el Protocolo de Brasilia y su mecanismo de solución de la controversia. Aunque el protocolo de Brasilia ha sido revocado por el protocolo de Olivos, que aborda la misma materia, los cuestionamientos levantados sobre estos temas continúan siendo de alta importancia para la necesidad permanente de la mejora del sistema jurídico de MERCOSUR, que exige la institucionalización de un tribunal de la justicia al ejemplo del europeo o del andino.

PALAVRAS-CHAVE: Direito da integração. Protocolo de Brasilia. MERCOSUL. Tribunal de justiça do MERCOSUL

KEY-WORDS: Right of the integration. Protocol of Brasilia. MERCOSUR. MERCOSUR's tribunal of justice.

PALABRAS-LLAVES: Derecho de la integración. Protocolo de Brasilia. MERCOSUR. Tribunal de justicia del MERCOSUR.

\section{Introducción}

El Protocolo de Brasilia para la Solución de las Controversias entre los Estados del Mercosur, primera disposición adoptada por el $\mathrm{CMC}^{1}$, entró en vigencia recién a partir de abril de 1993. Desde esta fecha hasta 1999 en ninguna oportunidad se habían completado las etapas para llegar a la sentencia arbitral que culmina el procedimiento regulado por dicho instrumento. 


\section{¿Es posible sostener que la integración en el Mercosur se desarrollaba} en forma tan equilibrada que no había sido preciso usar del sistema de Brasilia?. Tal vez ingenuamente podría sostenerse una respuesta afirmativa.

Bien es cierto que gran parte de los "roces" entre los socios (Estados miembros) se habían canalizado y solucionado a través del mecanismo de consultas y respuestas que se estableció en el ámbito de la $\mathrm{CCM}^{2}$. Sin embargo la solución final de cada controversia era (es) consensuada luego de negociaciones cuyo resultado si bien desde el punto de vista político no merecían (merecen) reproche, no sucedía (sucede) lo mismo cuando el análisis lo era (es) desde lo jurídico.

E1 procedimiento instituido por el Protocolo de Brasilia fue reglamentado a través de la Dec. $\mathrm{N}^{\mathrm{a}} 17 / 98^{3}$ (RPB).

Finalmente en 1999 el Mercosur tuvo la oportunidad de experimentar el uso, en dos oportunidades, del mecanismo de resolución de los conflictos, adoptándose dos laudos a través del Tribunal $A d H o c$ (TAHM), en los conocidos asuntos sobre restricciones no arancelarias ${ }^{\sharp}$, primero, y sobre subsidios a la exportación de productos destinados al comercio regionaf́, en segundo término; culminando el resumen "jurisprudencial" con la sentencia arbitral de marzo de 2000 en el caso de las salvaguardias al comercio intrabloqué.

Por obra de la mera casualidad (?), luego de estos tres ejercicios del mecanismo de Brasilia, los Estado miembros (EM) han decidido el "perfeccionamiento" del mismo en el marco de la conocida etapa de "Relanzamiento del Mercosur". Esta intención se plasmó en la Dec. № $25 /$ 00, adoptada durante la XVIII Reunión del CMC (Buenos Aires, 29/V/00) ${ }^{7}$. Por esta Dec. se delegó en el Grupo Ad Hoc de Aspectos Institucionales (GAHAl), la elaboración de una propuesta definitiva sobre la modificación del

\footnotetext{
2Dirs. CCM N $13 / 95$ y $6 / 96$

"Mercosur/CMC/DEC. N $17 / 98$ "Reglamento del Protocolo de Brasilia para la Solución de Controversias" (XV CMC, Río de Janeiro, 10/XII/98)

TAHM asunto 1/99, Cominicados $N$ 37/1997 y $N$ N 71998 del Depantanento de Operaciones de Comercio Exterior (DECEX) de la Secretaria de Comercio Exterior (SECEX). Aplicación de Medidas Restrictivas at comercio reciproco, 28/TV/99. Publicado en RDM 1999-4, pgs. 257-278.

TAHM, asunto 2/99, Subsidios a la producción y exportación de came de cerdo, 27/1X/99. Publicado en LL del $10 /$ XII/99, pg. 23.

"TAHM asunto 1/00, Aplicación de Medidas de Salvaguardia sobre productos Textiles (Res. 861/99) del Ministerio de Economia y Obras y Servicios Públicos (MEOySP), 10/III/00. Publicado en RDM 2000-3, pgs. $138-164$.

${ }^{7}$ Mrocosur/CMC/Drc. N2 $25 / 00$ "Perfeccionamiento del Sistema de Solución de Controversias del Protocolo de Brasilia". Su texto puede verse en BO $25 / \mathrm{NII} / 2000$, pgs. 30ss.
} 
Protocolo, la cual tendría que ser presentada antes del 10 de diciembre de 2000 .

El proyecto del GAHAI debia contemplar particularmente, entre otros temas, las cuestiones siguientes (art. 2):

a) el perfeccionamiento de la etapa posterior al laudo arbitral, en especial en lo que hace a su efectivo "cumplimiento", y el "alcance" de las medidas compensatorias que podrían aplicarse en su defecto.

b) parámetros fijos para la constitución de las listas de expertos y árbitros, y para su designación en cada caso.

c) mayor "estabilidad" de los árbitros.

d) opciones para lograr una "interpretación uniforme" de la normativa Mercosur; y por último,

e) mayor agilidad en los procedimientos existentes e implementación de procesos más expeditos para casos determinados.

Interin vencía el plazo se realizaron reuniones constante en el ámbito del GAHAI a fin de presentar en la fecha fijada el texto definitivo del "Proyecto de Reformas" al Protocolo de Brasilia (PRPB).

Cumpliendo en parte su tarea, el GAHAI presentó a la mesa de negociaciones un Proyecto que finalmente fue remitido al CMC para su aprobación, la cual se estimaba iba a suceder durante la Cumbre Presidencial de Florianópolis (XIX Reunión del CMC, 14-15 de diciembre de 2000).

A pesar de las arduas negociaciones el instrumento modificatorio recibiría el veto de la delegación uruguaya, el cual se basó en diversos motivos".

El resultado de la Cumbre, en lo que hace al tema en cuestión, fue la adopción de la Dec. $65 / 00^{\circ}$. Básicamente, lo que la norma establece es:

a) prorrogar el plazo establecido en la Dec. 25/00 hasta la próxima Reunión del CMC (art. 1); la cual tendrá lugar en Asunción, a mitad del corriente año.

b) crear un Grupo de Alto Nivel para la elaboración de la propuesta definitiva, la cual deberá tomar "en consideración el trabajo realizado hasta el momento" por el GAHAI y las "propuestas presentadas" por los EM (arts. 2 y 3 )

\footnotetext{
${ }^{8}$ Entre otras razones la delegación oriental -en armonia con la posición que siempre mantưo- exigia (a) una modifcación nảs profunda del sistema de Brasilia; (b) la constitución de un Trbunal Arbitral permanente. a cual pudierm tener acceso no sólo los EM sino también los particulares y (c) la creación de una Secretaria General que vigilara las medidas adoptadas por los EM a fin de dar cumplimiento al derecho Mercosur. No esta demas recordar que fueron los representantes uruguayos quienes presentaton a la Cumbre Presiden. cial de Ouro Preto (Brasil, 17 de diciembre de 1994) un Proyecto para la Creación de un Tribunal de Justicia para el Mercosur, elaborado por la Comisión Nacional de Juristas.

"Mercosur/CMC/Dic. N" 65/00 "Perfeccionamiento del Sistema de Solucion de Controversias del Protocolo de Brasila". Su texto puede verse en $\mathrm{BO} 1 / \mathrm{LI} / 200 \mathrm{\alpha}$, pgs. $11 \mathrm{ss}$.
} 
c) y finalmente, que entre las propuestas a ser elaboradas por el Grupo de Alto Nivel se "incluirá... la creación de un Tribunal Arbitral para el Mercosur".

Más allá del texto de la Dec., los EM lograron consenso sobre (i) la eliminación de la etapa que se realiza ante el GMC; (ii) la elaboración de una lista específica de Presidentes de los Tribunales Ad Hoc; (iii) las disposiciones sobre la fase posterior al laudo, esto es su ejecución y el alcance de las medidas compensatorias que podrían ser tomadas ante el incumplimiento del mismo; y (iv) -tal cual lo impulsara la delegación argentina- el establecimiento de un Tribunal Arbitral de Revisión (o de Segunda Instancia). Sobre este último ítem quedó “abierta" la cuestión de la composición del Tribunal de Alzada.

Como se ve la Dec. 65/00 no procede a derogar la Dec. 25/00, sino que sólo amplía el plazo que ésta determinó, con lo cual el Proyecto a ser presentado deberá contemplar -en forma obligatoria- los criterios de revisión enunciados en la Dec. 25/00.

Debe destacarse que resultó "expresa" la consideración según la cual esta "pequeña reforma" no significaría dar cumplimiento al mandato que establece el art. 44 POP, a saber que antes de la culminación del proceso de convergencia al Arancel Externo Común [diciembre de 2005] "los Estados Partes efectuarán una revisión del actual sistema de solución de controversias del Mercosur con miras a la adopción del sistema permanente" (Cfr. también art. $34 \mathrm{~PB}$ )

\section{El Proyecto de Reformas al Protocolo de Brasília (PRPB)}

1 - Através de la modificación del art. 4 PB se elimina, como regla, la intervención del GMC, como etapa posterior en los supuestos en los que las negociaciones directas no hubieran tenido una solución satisfactoria.

De cualquier manera los Estados involucrados en el conflicto pueden de común acuerdo optar por la intervención del GMC. Lo mismo ocurre cuando otro Estado ajeno a la reclamación solicita, en forma fundada, que se planteé el asunto ante dicho órgano; sin embargo, esta circunstancia no afectará la continuación del procedimiento arbitral, salvo acuerdo en contrario de todos los Países involucrados.

2- Se altera también el art. 10 PB (y consecuentemente, el art 9) al insertarse dos nuevos párrafos a través de los cuales se suma a la Lista originaria de Árbitros por Estados (párrafo primero) una "Lista Específica 


\section{de Terceros Árbitros" (Presidentes de los TAHM) que se conformará} a partir de la designación de tres árbitros por cada País; uno de los integrantes de esta última lista no podrá ser nacional de los Estado Parte del Mercosur. Ambas Listas (la fijada en el art. 10 actual y la incorporada por el Proyecto) serán sometidas a la consideración de los demás EM, quienes a su vez podrán realizar, en el plazo que se establece, objeciones justificadas. No se ha pactado acerca de los criterios, la naturaleza, las modalidades y los efectos de la eventual objeción.

En los casos en que los Estados parte en la controversia no llegaren a un acuerdo sobre la designación del Árbitro Presidente (art. 12), la misma recaerá, a pedido de alguno de estos Estados, en la SAM, la que hará la elección por sorteo, en base a la Lista Especifica excluyendo a tal fin los nacionales de los Países involucrados en el pleito.

3- Otra modificación importante es la "constitucionalización" de las exigencias sobre la "necesaria independencia" e "imparcialidad" que deberán ser observadas por los árbitros de ambas listas. Hacemos referencia a la constitucionalización debido a que estos criterios ya estaban fijados en el Derecho derivado, en concreto en el art. 16 RPB (esta norma sólo establece estos deberes para los árbitros "designados" en un caso concreto). Con la nueva redacción del art. 13 PB dichas exigencias adquieren el rango de Derecho originario o Constitucional del Mercosur ${ }^{10}$.

Lo mismo sucede con el mandato contenido en la Dec. 28/94 ${ }^{11}$.

4- En cuanto a las reglas de procedimiento del TAHM, el PRPB limita la discrecionalidad que tienen los árbitros en cuanto a su elección (arts. 15 PB y $20 \mathrm{RPB}$ ), determinando que éstas serán adoptadas "con base en las Reglas Modelo a ser aprobadas por el $C M C^{12}$.

5- Sin dudas una de las modificaciones más llamativa es la que se refiere a la institucionalización de un Tribunal Arbitral de Revisión (TAR; art. 21 PB reformado). El mismo estará compuesto sólo por tres árbitros "designados a partir de las listas nacionales de tres árbitros indicados por los Estados Partes de común acuerdo".

\footnotetext{
${ }^{10}$ A diferencia del RPR, que prescribe el deber de independencia e imparcialidad de los árbitros no sólo respecto de los "Estados parte en la controversia" sino también con relación a "terceros", el Proyecto únicamente menciona a "los Gobiemos de los Estados Partes" (es decir, también respecto de aquellos no parte en el conflictol.

"Mercosur/CMC/DrC. N"28/94 "Tribunais Ad Hoc do Protocolo de Brasilia"; ait. 1 "Os Tribunais Arbitrais Ad Hoc, a que se refere o artigo $8^{\circ}$ do Protocolo de Brasilfa, terão como sede a cidade de Assungäo".

${ }^{12}$ Dichas reglas están siendo elaboradas por el GAHAI y esta previsto que el Documento final sea elevado en el corto plazo a la consideración del CMC para su aprobacion.
} 
Los integrantes del TAR tendrán "carácter estable", es decir permanente; lo que queda garantizado además a través de la exigencia según la cual los "criterios de sustitución" de los mismos deberán ser "estrictos" (art. 21 $\mathrm{PB}$ reformado). No se establece sin embargo la duración del cargo de los magistrados del TAR, ni los criterios de sustitución. Tampoco se hace referencia a la nacionalidad que deberán tener los árbitros.

Debe destacarse que, combinando su composición numérica y la cantidad de Estados del Mercosur, el TAR tendría un carácter aún más "comunitario" o "supranacional" que otros órganos de solución de diferencias, en materia de integración. Así por ejemplo, el Tribunal de Justicia de las Comunidades Europeas (TJCE) está constituido por igual número de jueces que EM (TJCE: 15 magistrados); lo mismo cabe precisar con respecto al Tribunal de Justicia de la Comunidad Andina (TJCA: 5 magistrados). No ocurre así en el caso de la actual Corte Centroamericana de Justicia (CCI) la cual si bien esta compuesta por seis magistrados, éstos son elegidos, en número de dos, por los Países respecto de los cuales están vigentes tanto el Protocolo de Tegucigalpa, como el Estatuto de La Corte (es decir El Salvador, Honduras y Nicaragua; no así para Costa Rica, Guatemala y Panamá, países que, al igual que los primeros, forman parte del SICA en cuyo marco tiene jurisdicción la CCI).

El recurso ante el TAR -que carece de efecto suspensivo en lo que hace al cumplimiento del laudo ${ }^{13}$ - deberá ser interpuesto dentro del plazo de quince días contados a partir de la notificación del laudo ${ }^{14}$. En cuanto a la materia sujeta a revisión el proceso semeja un Tribunal de casación arbitral, toda vez que únicamente las "cuestiones de derecho" y las "interpretaciones jurídicas" -desarrolladas en la decisión atacada- serán susceptible de ser mantenidas, modificadas o revertidas por el TAR.

6- También es oportuno resaltar los cambios que se introducen en lo que atañe a la ejecución del laudo y al alcance de la eventual aplicación de medidas compensatorias.

En cuanto a lo primero, el PRPB tiende a una "relativa comunitarización del cumplimiento del laudo" al establecer que el Estado vencido deberá -en el término de cinco días, contados a partir de concluido el plazo

\footnotetext{
1. Llama la atencion que no se haya fijado, dentro de la competencia del TAR, la posibilidad de dictar "las medidas provisionales que considere apropiadas" (dentro de las cuales podría caber la de suspender la ejecución del laudo), que si están dentro de las facultades del Tribunal Arbitral de primera instancia (Cfr. arts. $18,21.4$ y 22.3 PB reformado).

"Aín esta abierto a la negociación si el recurso de revisión sólo será susceptible de recaer sobre los puntos en los cuales no haya existido unanimidad o si podrá versar sobre todos los ítems de la sentencia impugnada.
} 
para su ejecución- informar tanto a la contraparte como a los Coordinadores Nacionales del GMC "sobre las medidas adoptadas" para la implementación del mismo (art. 22 PB reformado).

De presentarse alguna divergencia en cuanto a estas medidas (sólo) el Estado reclamante podrá requerir -vía SAM y en el término no mayor a los treinta días de finalizado el plazo para el cumplimiento del laudo la constitución del Tribunal original que entendió desde el inicio en el conflicto, a fin de que se expida al respecto. Dicha convocatoria no tendrá efectos suspensivos sobre la ejecución del laudo, salvo consideración en contrario del Tribunal (art. 22.3 PB reformado).

Uno de los puntos más "candente" en el actual sistema de Brasilia lo constituye sin dudas la cuestión de las compensaciones en caso de incumplimiento de los laudos. Al respecto, el Proyecto fija que dichas medidas sólo podrán ser aplicadas dentro de un "plazo máximo", el cual debería ser establecido considerando, entre otros factores, el cumplimiento del laudo, el perjuicio ocasionado y los acuerdos a los que arriben las partes.

Como última posibilidad de corrección de la conducta infractora se ha acordado que el País reclamante deberá dar aviso ( 15 a 30 días antes) al Estado reclamado sobre las medidas que proyecta ejecutar. Este último podrá, de considerarlas excesivas, plantear la convocatoria del Tribunal que entendió en el litigio a fin de que se expida sobre la divergencia; dicha facultad sólo podrá ser ejercida dentro de los quince días posteriores al anuncio de la aplicación de compensaciones hecha por el Reclamante, quien a su vez, deberá, de ser necesario, modificarlas de acuerdo al criterio que establezca el Tribunal reunido al efecto.

En cuanto a la tipología de acciones conminatorias (suspención de las concesiones/obligaciones o medidas equivalentes) que pueden adoptarse ante la falta de implementación del laudo el PRPB establece que el Estado demandante "procurarâ" que las mismas sean aplicadas en el mismo sector o sectores sobre los cuales se ha dictaminado el incumplimiento (el Proyecto menciona "sector o sectores afectados"), salvo que, en forma fundada, indique que esta opción sería "impracticable o ineficaz", supuesto en el cual podrá ejecutarlas "en otro sector" (art. 23.3 PB reformado).

\section{El Proyecto de Reformas y la Decisión 25/00 CMC}

a- En cuanto al mandato de la Dec. 25/00 sobre "agilización de los procedimientos" hoy vigentes, lejos de cumplirlo el Proyecto, si bien permite -a criterio de las partes- la eliminación de la intervención del GMC, por otra parte amplía los mecanismos al instituir el TAR, lo cual trae aparejado el alargamiento de los plazos finales.

También se han contravenido los términos de la Dec. 25/00 en 
cuanto esta exige que la propuesta de reformas incluya el establecimiento de "procedimientos sumarios" para supuestos concretos. Seguramente la intención de la Dec. tenga que ver con los casos de incumplimientos flagrantes o manifiestos del Derecho Mercosur. Una regulación en este sentido existe en el sistema procesal andino para las hipótesis de restricciones o gravámenes verificados y de violación flagrantes al Derecho comunitario ${ }^{15}$.

b- Si bien la reglamentación de la "etapa posterior al laudo" y de la cuestión de las "medidas compensatorias" también ocasiona un alargamiento final de los términos, la regulación era materia pendiente del Sistema de Brasilia razón por la cual estaría justificada la modificación.

En lo que hace al tema de la tipología de acciones compensatorias que el Proyecto permite, creemos oportuno resaltar lo siguiente. En los sistemas de Derecho procesal comunitario comparado se han fijado para los supuestos de incumplimiento de sentencias tres mecanismos diferentes:

-En la Comunidad Europea (CE), es la Comisión quien discrecionalmente puede optar por acudir al TJCE a fin de que éste, determinado que sea la falta de cumplimiento de la sentencia en cuestión, imponga al EM, el pago bien de una suma a tanto alzada bien de una multa coercitiva (228.2 CE). Al día de hoy esta acción sancionatoria ha sido ejercida sólo en una oportunidad ${ }^{16}$. Por razones que huelga mencionar, tal sistema sería de muy dificil implementación en el Mercosur, principalmente por la anemia institucional que aqueja al bloque.

-En el Sistema de la Integración Centroamericana (SICA), ante la hipótesis bajo análisis, el Estatuto de la CCJ se limita a fijar que el Tribunal meramente comunicará tal circunstancia a los otros EM a fin de que éstos, haciendo uso de los medios que crean pertinentes, aseguren

\footnotetext{
${ }^{15}$ Art. 26 Thatado de Creación del TJCA.

16TJCE: as, C-387/97, Comisión/Greda, 4/VI/00, Rec 1-0000. (condusiones del Abogado General, Sk. DAMAso RumJarago Colomer, presentadas el 28/TX/99; Rec I-noo0). La sanción que se impuso a Grecia, consistente an el pago de una multa coercitiva de 15.375 euros diarios (pagaderos desde la fecha de notificación de la sentencia hasta que se ponga fin a las infracciones), obedecio a la falta de ejecución de la sentencia de incumplimiento dictada en el as. C45/91, Comisión/Grecia, 7/V/92, Rec. 12509.

Cabe destacar además que la Comision interpuso un segundo procedimiento para la aplicacion de sanciones por incumplimiento de sentencia (as. C-197/98, Comision/Grecia, por inobservancia de la sentencia C-365/93, Comision/Grecia, 23/LII/95, Rec. [499). Dicho expediente, que contaba con las conciusiones del Abogado General (SR. FENner.w, presentadas el $9 / \mathrm{XII} / 99$, Rec. 10000), fue archivado por auto del Presidente del TJCE de $6 / \mathrm{X} / 00$ (Rec. I-0000), en razón del desistimiento de la Comisión, luego que Grecia procediera a poner en practica las medidas necesatias para dar ejecución al fallo incumplido.

A su vez la Comisión ha interpuesto otros procesos de sanciones los cuales se encuentran pendientes de resolución ante el TJCE -dos contra Francia y dos contra Grecia- [sobre esto úlimo, ver "XVII" Informe Anual sobre el Control de la Aplicación del Derecho Comunitario (1999)", Doc COM/2000/92 final, de 23/N//00].
} 


\section{la ejecución de su decisión (art. 39 Estatuto CCJ) ${ }^{17}$. Este mecanismo -que no} difiere en mucho del actualmente vigente en el Mercosur- tiene por un lado, la inconveniencia de poner en manos de los propios EM la elección, conveniencia y tipo de medidas a adoptarse, y por el otro, la ventaja de facultar a La Corte para investigar de oficio la efectiva ejecución de sus decisiones. -Por último, en la Comunidad Andina (CA) el art. 27 del Tratado Constitutivo del Tribunal de Justicia establece que si en el plazo de tres meses a partir de la notificación, el EM condenado no ejecuta la sentencia, el Tribunal de oficio y en forma sumaria, previa opinión de la Secretaria General, fijará los límites dentro de los cuales "cualquier Estado miembro podrá restringir o suspender total o parcialmente las ventajas del Acuerdo de Cartagena en relación con el país remiso" ${ }^{\prime 2}$. A su vez, si tales acciones agravan la situación o se presentan como ineficaces, el Tribunal podrá también "ordenar la adopción de otras medidas".

\footnotetext{
${ }^{17}$ En la práctica La Corte ha conocido en un caso de incumplimiento de sentencia (inobservancia de su Resol. de 17/ III/97, Sra. Jearette del Carnen Vega Baltodano contra el Poder Ejecutivo de Nicaragua s'dematada de incumplimiento de hudo arbitral, GOCOJ Na $5,1 / 2 / 97, \mathrm{pg}$. 2) ante lo cual resolvió comunicar a los demás EM el incumplimiento de su sentencia por parte de Nicaragua, a fin de que $\epsilon$ tos «uthizando los medios pertinentes, aseguren su ejecuciono [Cfr. Resol. de 5/[X/97, Sra. Jeanette del Carmen Vega Baltodano (II), GOCCJ N² 6, 25/[X/97, pg. 13 (SCIV)] A su vez La Corte ha desestimado, al menos por el momento, la pretensión de Honduras a fin de que se declare que Nicaragua ha incumplido con la medida cautelar dictada por el Tribunal [caso Honduzas/Nicaragua (Incumpliniento de aormas comunitarias), 12/1/2000, GOCC) N=10, pg. 9] por la cual se ordenaba a este útimo la suspersión de la aplicación de la Ley $\mathrm{N} 325$ ("Ley creadora de impuesto a los bienes y servicios de procedencia u origen hondureno y colombiano": arancel del 35\%) y consecuentemente se comunique a los Estados del SlCA tal contravención a fin de que se tomen las medidas pertinentes para la ejecución del fallo, debido a que del sonjunto la documentación que ha presentado como prueba el Estado de Fonduras, no se encuentra documento alguno que compuebe tal situacionn [Cfr. caso Honduras/Nicaragua foncumplimiento de normas comunitanas) 26/[X/2000]. La decisión contó con el voto disidente del MAG. ADoLro LEon Gowez. ${ }^{15}$ E1 TJCA ha iniciado tres procedimiento (sumarios) para la aplicacion de sanciones, a saber: (a) ssumario por incumplimiento de sentencia, proc, 1-AI-97, funta/ Wenezuela, auto del 20/X/99, GO Na 500, 25/X/99 Por la inobservancia de Venezuela, de su obligación de eliminar las restricciones (sanitarias) a las importaciones de aio provenientes de Perú, tal como lo ordenaba la sentencia del proc. 1 Al-97, Junta/Venezuela, 11/XI/97, GO Na $329,9 / \mathrm{III} / 98$, (b) ssumario por incumplimiento de sentencia, proc. 1-A196, Junta/Ecuador, auto del $28 / \mathrm{VIV} / 99$ GO N: $508,15 / \mathrm{XI} / 99$ [Por la reiterada actitud de Ecuador de mantener en vigor y aplicar disposiciones axcionales que vulneran el sistema andino sobre patentes (Decisión 344), incumpliendo la sentencia del proc. 1 .

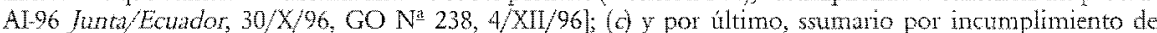
sentencia, proc 3-AL-96, Junta Nenezuela, auto del $27 / \mathrm{X} / 99, \mathrm{CON} 512,26 /$ XI/99 [Por la reticencia de Venezud a suprimir los obstáculos sanitarios a las importaciones de cafe tostado proveniente de Colombia, tal como era su obligación a tenor de la sentencia del proc. 3-AI-96, Junta/lenezuela, 24/II//97, GO Na 261,29/N/97].

En los casos concretos, se decretó que, hasta tanto el pás correspondiente demostrara fehacientemente ante Tribunal, el cabal cumplimiento de las sentencias el testo de los EM suspenderian las ventajas del Acuerdo de Gartagena que al momento lo benefician, y autorizó a los respectivos Gobiernos a: i) la imposición de un gravamen adicional del 5\% a las importaciones, originarias de Venezuela, de hasta cinco de los productos de mayor volumen de comercio sumario en el proc. 1-A[-97. Por auto del 10 de noviembre de 2000 -GO Na $621,30 / \mathrm{XI} / 00$ el Tribunal puso fin a las sanciones por clianto el Gobierno de Venezuela dio efectivo cumplimiento a la decisión judicial); $i$ li la reducción de las preferencias ecuatorianas en materia de ontren del 60 al 50\% (sumario en el proc. 1-AL-96); y in) la imposición de un gravamen adicional del 50\% a has importaciones de dos productos del sector agropecuario a su elección, originarios de venezuela (sumario en el proc. 3-A1-96. Por auto de 25 de febrero de 2000 el Trbunal dejó sin efecto la sanción que pesaba sobre Venezuela en razon de haber cumplido dicho Paśs con sus obligaciones).

"Ver también el "Reglamento Especial sobre sumarios por incumplimiento de sentencias", aprobado por el Tribunal de Justicia el 7 de mayo de 1999 (GO Na 455, 22/VI/99).
} 
Esta alternativa -muy similar a la que intenta implementar el PRPB-ha sido criticada por la propia doctrina andina debido a que las consecuencias que ella acarrea para el proceso de integración en su conjunto son más perjudiciales que el desacato que se intenta subsanar. En efecto, a través de estas medidas lo que se logra en el fondo es retroceder en el programa de liberalización comercial, pues se deja fuera del sistema al País remiso, y por consecuencia, en vez de profundizarse en la liberalización de los intercambios intrabloque -meta muy dificil de consolidar- se retrocede hacia la partición del mercado ampliado. Con estas medidas, la integración ocasiona menos integración.

Si acaso, cabría la posibilidad de analizar la compatibilidad de sanciones alternativas. Así por ejemplo, no debería negarse, a priori, la conveniencia de establecer como medida para obtener la ejecución del laudo, la "suspensión del derecho a voto del Estado remiso en los órganos del Mercosur".

La opción que planteamos para debate podría estar redactada de la siguiente manera:

"Protocolo de Brasilia reformado, art. 23:

(párrafo primero) Si un Estado parte no cumpliere el laudo del Tribunal Arbitral en el plazo de treinta (30) días, los otros Estados partes en la controversia podrán optar, en forma alternativa, por las medidas a que se refieren los párrafos segundo o tercero.

(párrafo segundo) En el supuesto contemplado en párrafo anterior, el Estado parte incumplidor, no podrá ejercer, a partir de la próxima Reunión Cumbre de Presidentes del Mercosur inclusive y hasta tanto implemente efectivamente el laudo, su derecho a voto en todos los órganos del bloque que corresponda, salvo cuando la reunión aludida se llevare a cabo dentro de los veinte (20) días posteriores al vencimiento del plazo de treinta (30) dias mencionado en el párrafo primero, supuesto en el cual la sanción comenzará a regir, en iguales términos, a partir de la siguiente Reunión Ordinaria del Grupo Mercado Común. La ausencia de voto así determinada no impedirá a los órganos correspondientes la adopción de normas obligatorias para todos los Estados Parte.

(párrafo tercero) (se mantendría la igual redacción que aparece en el Proyecto de Reformas como párrafos primero y siguientes)... En el supuesto contemplado en el párrafo primero, los otros Estados partes en la controversia podrán adoptar medidas compensatorias temporarias (...)"20.

Esta forma de sanción también se encuentra regulada en la Comunidad Andina para los supuestos en los que los EM acumulen un retraso de un año en el depósito de los fondo correspondientes al presupuesto de la Secretaría General o del TJCA2.

\footnotetext{
Articulo suieto a posterior reglamentacion.

${ }^{2}$ Ant. 28, Acuetdo de Cartagena (codificado mediante Decision No 406, GO N 277, 4/VI/97): "El Pals Membo que incurriere en un retraso mayor a cuatro trimestres en el pago de sus contribuciones corrientes

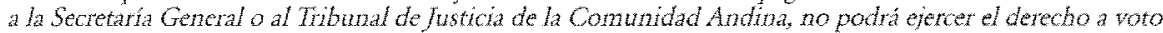
an la Comision hasta tanto regularice su stuacion".
} 
De esta forma se evitarian las críticas que se han presentado en el sistema andino con respecto a las medidas compensatorias.

c- Por otro lado el Proyecto avanza en la exigencia de "mayor estabilidad de los árbitros" (Dec. 25/00), lo cual se materializa principalmente en la implementación y composición tanto de la Lista Específica de Terceros Árbitros-Presidentes como del Tribunal Arbitral de Revisión.

d. En nuestra opinión la reforma no cumple otro de los criterios de revisión fijados en la Decisión mencionada; si acaso uno de los más importantes, cual es la elaboración de mecanismos para lograr la "interpretación uniforme" del Derecho Mercosur. Obviamente no basta a tal fin con la Lista Especifica de Terceros Árbitros-Presidentes o con el Tribunal Arbitral de Revisión.

No hubiera sido para nada desacertado que el GAHAI hubiera implementado un mecanismo similar a la conocida "cuestión prejudicial" (de interpretación $y / o$ validez) a fin de otorgar una herramienta muy valiosa al juez nacional que se enfrenta, cada día con mayor frecuencia, ante la "novedad" de tener que aplicar un sistema normativo (Derecho Mercosur) autónomo claramente diferenciado tanto del ordenamiento jurídico nacional como internacional. Cabe recordar que el mecanismo de la cuestión prejudicial ha sido el método de mayor eficacia en los procesos de integración comparados a fin de lograr la uniformidad en la interpretación y aplicación del Derecho comunitario ${ }^{22}$.

e- A su vez el PRPB avanza muy tímidamente sobre la elaboración de "criterios" para la designación de los expertos y de los árbitros para cada caso.

En realidad la idea de la designación de los árbitros para cada caso, o en otras palabras la naturaleza ad hoc del Tribunal Arbitral del Mercosur, es un innegable atentado contra la exigencia de la uniformidad en la interpretación y aplicación del Derecho de la integración; y esto por razones obvias, baste por el momento comparar los tres laudos arbitrales que se han dictado a la fecha para confirmar lo que se viene considerando.

La opción acertada, en nuestra opinión, lejos de ser la de constituir Tribunales arbitrales especializados, es decir conformados de acuerdo a la materia del conflicto, sería establecer un Órgano Jurisdiccional permanente para todos los conflictos a fin lograr así líneas jurisprudenciales coherentes, no contradictorias ${ }^{23}$, que refuerce el ordenamiento jurídico

\footnotetext{
${ }^{22} \mathrm{CF}$, art. $234 \mathrm{CE}$; CAN: arts. 3236 Tratado de Creación del TJCA; SICA: art 22.k Estatuto CCJ (Cfr. también art. $22 . d$ y 23 ).

"Sólo para poner un ejemplo de jurisprudencia contradictoria entre los TAHM que han intervenido recomendamos analizar los diferentes criterios utilizados en el Segundo y Tercer Laudo a la hora de la "determinación del objeto de la controversia".
} 
regional. Se acepte o no, más allá de aplicar el Derecho Mercosur al caso concreto planteado, cada Tribunal arbitral realiza un ejercicio de profundización y afianzamiento del sistema normativo; circunstancia que pierde casi toda su transcendencia y valor en razón del carácter ad hoc del mecanismo hasta hoy vigente.

\section{Consideraciones globales acerca del Proyecto de Reformas}

i) La primera crítica que es posible hacer al Proyecto se refiere al completo hermetismo en que se desarrolla su elaboración y negociación. Lo cual adquiere mayores contornos si se repara en la "crucial" importancia que todo sistema de solución de controversias tiene sobre el proceso de integración. Tal vez hubiera sido altamente recomendable que esta cuestión se presentara al debatey la discusión de la opinión pública y en especial de los sectores privados (cámaras industriales y de comercio) y académicos vinculados al Mercosur (universidades).

ii) Más allá de la inconveniencia de introducir en el mecanismo un nucvo Tribunal arbitral de apelación o revisión, si consideramos aspectos tales como su composición (número de integrantes, estabilidad, etc.) tal vez sería éste el camino para lograr que algunos EM pierdan el "temor" que han demostrado hasta el momento en lo que hace a la creación de un verdadero Tribunal de Justicia del Mercosur.

iii) Continúa la actitud de algunos Gobiernos en el sentido de negar cualquier acceso de los particulares a la justicia en el Mercosur, a través de un recurso directo, ya sea por acción de incumplimiento contra los EM, o por medio del mecanismo de la cuestión prejudicial, o vía acción de nulidad contra las normas del bloque. Esto sin dudas no se compatibiliza, bajo ningún punto de vista, con el verdadero papel de los particulares dentro del mercado, si se considera -como es- que su cotidiana actividad, a través de los intercambios comerciales, constituye, en gran medida, el "motor" de la integración en el bloque.

\section{Anexo: Sobre el Mito de la innecesatiedad de un Tribunal de Justicia del MERCOSUR}

Para concluir nos referiremos a un argumento que se utiliza con la finalidad de demostrar la falta de necesidad de un Tribunal de Justicia para nuestro bloque. Lo grave del mismo no radica sólo en que es 
repetido por algunos funcionarios de los Gobiernos encargados de las negociaciones en el seno del Mercosur, sino que también es defendido por importantes doctrinarios versados en temas de integración ${ }^{24}$.

Suele afirmarse que la constitución de un Tribunal de Justicia no sería adecuada ni aún necesaria en la etapa actual del Mercosur, ya que o bien el comercio entre los socios no es todavía de gran magnitud, o bien el número -por ahora- exiguo de conflictos planteados no justificaría la institucionalización de un órgano permanente.

Veamos si el recurso a la experiencia comparada avala esta construcción. Para ello recurriremos al ejemplo del TJCE, del TJCA y de la CCJ, ya que los procesos de integración en los cuales actúan estos órganos jurisdiccionales guardan gran analogía con el Mercosur.

\section{- ¿Las Comunidades Europeas, a la hora de instaurar el Tribunal de} Justicia, esperaron a que el volumen de los negocios en el interior del bloque alcanzara un índice elevado, o a que los conflictos intracomunitarios se plantearan en un número importante?

Ciertamente, tanto la CECA, por un lado, como la CEE y la CEEA, contaron precisamente desde el primer día de entrada en vigencia de sus Tratados constitutivos respectivos con una Corte de Justicia. Es decir aún antes de que se efectuara intercambio comercial alguno o que se planteara conflicto cualquiera.

¿Cuál es el mecanismo que ha posibilitado la mayoría de las sentencias de los Tribunales referidos?

Sin dudas la respuesta no se presta a debates: el mecanismo en cuestión es la consulta prejudicial (de validez e interpretación - TJCE; sólo de interpretación - TJCA y CCI). En efecto, en el recuento de procesos ante estos Tribunales, el reenvio prejudicial -salvo en el caso de la $\mathrm{CCJ}^{25}$ ocupa claramente y por lejos el primer lugar.

\footnotetext{
${ }^{24}$ Entre otros, BAPTISTA, Luiz Olavo, (arbitro del II" laudo del TAHM), "Soluça de drvergências no Mercosul", en Mercosul. Seus efeitos jutrdicos, econômicos e politicos nos Estados membros (Maristela Basso, organizadora), 2da. ediçăo, ed. Líbrería dos Advogados, Porto Alegre-RS-1997, pgs. 178-186 (157/186); FÉRNADEZ REYES, Jorge E. (ex Director de la SAM), "Evaluación de los mecanismos de solución de controversias en el Mercosur", RDM 2000-4, pgs. 159-163 (139-169): GONCALVES, José Botafogo, "Os Tribunais do Mercosu1". Boletim de Integraçio Latino-Americana $\mathrm{N}^{ \pm}$18, janciro-junho/1996, ed. MREGIEGETEC, Busilia, pg. 677; SOARES, Guido Silva (integrante de la lista de ábitros del Mercosur), "No es momento para una mayor institucionalización del bloque", entrevista realizada por Garriela Wurcer, RDM 2000-3, pg. 36 (35-36).

${ }^{25}$ La razón bien podrín radicar, entre otras razones, en que, por un lado, en el sistema centroamericano -a diferencia de lo que sucede en los ejemplos europeo y andino- no se ha regulado en ningún supuesto la obligación de realizat la consulta prejudicial de interpretación, adenaás, por el otro, debe resaltarse que La Corte ha iniciado sus actividades recién el 12 de octubre de 1994.
} 
Aún más. Los verdaderos leading cases de estos Tribunales han sido establecidos en el marco de los procesos prejudiciales. Sólo para citar algunos ejemplos, pueden recordarse, en la CE los precedentes Van Gend \& Loos, Costa/ENEL, Stauder, Internationale, Simmenthal I, CILFIT, Foto-Frost, Zuckerfabrik, Factortame, Francovich ${ }^{26}$; en la CA las sentencias Aktiebolaget Volvo, Cavelier, Cavelier y Vernot, Nike International, Nombres de publicaciones periódicas y de programas de radio y televisión, Convenio de París para la protección de la Propiedad Industrial, ACEGRASAS, Cigarrera Bigott, Cavelier Gaviria ${ }^{27}$.

Por otro lado este procedimiento asegura -aún cuando sea de modo indirecto- el acceso del particular a la administración de justicia comunitaria. No deberíamos profundizar demasiado para llegar a la conclusión de que en el Mercosur el derecho fundamental de la persona a la tutela judicial efectiva es el gran ausente en el proceso de integración. -¿Qué conclusión es posible extraer respecto a la posibilidad de que los EM se demanden recíprocamente en los supuestos de incumplimiento de la normativa comunitaria?

Basta a este respecto revisar los repertorios de jurisprudencia de todos y cada uno de los Tribunales de Justicia citados para llegar a la siguiente conclusión: los Estados miembros de un proceso de integración son totalmente esquivos a interponer demandas contra otro Estado parte en las hipótesis de incumplimiento. A sólo manera de graficar esta afirmación, veamos la siguiente comparación:

-En la CE: en casi cincuenta años de integración únicamente en dos oportunidades se ha llegado a sentencias de Tribunal a partir de procesos iniciados por un EM; fueron los casos Reino Unido/Francia (1979) y Bélgica/España (2000)28.

\footnotetext{
26TJCE: as. 26/62, Van Gend y Loos, 5/II/63, Rec. Selección 1961-1963, Luxemburgo, 1999, pg. 333; as. 6/64, Costa/ENEL, 15/VII/64, Rec. Selección 1964-1966, Luxemburgo, 1999, pg. 99; as. 29/69, Stauder, 12/XI/69, Rec. 1969 pg. 419; as. 11/70, Internationale Handelsgesellschaft mbH, 17/XII/70, Rec. Selección 1970, Luxemburgo, 1999, pg. 241; as 106/77, Simmenthal I, 9/III/78, Rec. 1978 pg. 629; as. 283/81, CILFIT, 6/X/82, Rec. 1982 pg. 3415; as. 314/85, Foto-Frost/Hauptzollamt Lübeck-Ost, 22/X/87 Rec. 1987 pg. 4199; ass, acs. C143/88 y C-92/89, Zuckerfabrik Süderdithmarschen $A G, 21 / \mathrm{II} / 91$, Rec. I-415 (\$16-21); as. C-213/89, Factortame y otros, 19/VI/90, Rec. I-2433; ass. acs. C-6 y C-9/90, Francovich y otros, 19/XI/91, Rec. I-5357.

${ }^{27}$ TJCA: proc. 1-IP-87, Aktiebolaget Volvo, 3/XII/87, GO Na 28, 15/II/88; proc. 2-IP-88, Cavelier, 25/V/88, GO Na 33, 26/VII/88; proc. 2-IP-90, Cavelier y Vexnot, 20/IX/90, GO Na 69, 11/X/90; proc. 3-IP-90, Nike International LTDA, 25/IX/90, GO Na 70, 15/X/90; proc. 10-IP-94, Nombres de publicaciones periódicas, programas de radio y televisión y estaciones de radio difusión, 17III/95, GO № 177, 20/IV/95; proc. 1-IP96, Convenio de París para la protección de la Propiedad Industrial, 9/XII/96, GO Na 257, 14/IV/97; proc. 30-IP-98, ACEGRASAS, 16/VI/99, GO N ${ }^{2}$ 475, 1/IX/99; proc. 15-IP-99, Cigarrera Bigott, 27/X/99, GO N 528, 26/1/00; proc. 64-IP-2000, Cavelier Gaviria y ottos, 6/IX/00, GO Nª 602, 21/IX/00.

${ }^{28}$ TJCE: as. 141/78, Francia/Reino Unido, 4/X/79, Rec. 1979, pg. 2923; as. C-388/95, Bélgica/España, 16/V/00, Rec. I-3123. Se han incoado otros procedimientos iniciados por Estados miembros contra otros Estados miembros, sin embargo fueron luego archivados por distintas razones: cfrs. as. 58/77, Irlanda/Francia, archivado el 15/II/ 78; as. C-349/92, España/Reino Unido, archivado el 27/XI/92.
} 
-En la CA: pueden hacerse las mismas consideraciones, aún también en lo que hace al número de asuntos planteados (Venezuela/Ecuador caso Belmont- y Venezuela/Colombia, ambos de $1997^{29}$ ).

-En el SICA: con la reserva de la exigua vida de La Corte (6 años a estos días), cabe expresar que la demandabilidad entre los EM es también una excepción: dos supuestos hasta el momento (Nicaragua/Honduras -1999- y Honduras Nicaragua -2000-30).

Bien entendido que lo hasta aquí afirmado no significa que no se plantean procesos por violación del Derecho comunitario tanto en la CE como en la CA, sino que el grueso de estos procedimientos se inicia a partir de las presentaciones que realiza, en cada esquema de integración, el "órgano comunitario", es decir aquel cuya función no es representar a los EM sino velar por la salvaguarda del interés de la Comunidad, lo cual le posibilita que al no depender de directivas de los respectivos Gobiernos, pueda, en uso de su autonomía e independencia, requerir a los Tribunales respectivos la condena de la actitud infractora de los distintos Estados (Comisión, en la CE y Secretaría General, en la CA).

La ausencia en el Mercosur de un órgano con estas características impide que se planteen los casos de incumplimiento de los Estados. En efecto, al estar integradas todas y cada unas de las instituciones del bloque por funcionarios que siguen las directivas de los respectivos Gobiernos es imposible que exista un órgano especializado en vigilar a los Países a la hora de dar cumplimiento al Derecho Mercosur.

\section{Conclusión}

Para concluir, y responder al argumento referido al principio de este título, podemos considerar que la afirmación sobre la falta de necesidad de una Corte de Justicia para el Mercosur, al menos desde la perspectiva comentada (reducido margen -aún- de comercio intrabloque y/o escaso número de conflictos planteados) encierra un vicio circular.

\footnotetext{
${ }^{29}$ T/CA: proc. 2-A1-96, Venezuela/Ecuador (caso Belmont), 20, NI/97, GO Na 291, 3/LX/97; proc. 4.A1-96, Venezuela/Colombia, $16 / \mathrm{K} / 97, \mathrm{GO} \mathrm{N}^{2} 308,28 / \mathrm{XI} / 97$.

${ }^{3} \mathrm{CC}$; caso Nicaragua/Honduras (Incumplimiento de normas comunitarias), 30/X//99, GOCCf N¹0, pg. 7; caso Honduras/Nicaragua (Incumplimiento de nomas comunitarias), 12/1/00, GOCOJ N 10, pg. 9
} 
En efecto, no es acertado sostener que no se han presentado controversias que justifiquen un órgano jurisdiccional permanente, cuando, precisamente, el sistema que se ha instaurado para la solución de los conflictos impide, no por defectos sino por esencia y estructura, que sea posible incoar procedimiento alguno.

Sea por ausencia del mecanismo de las cuestiones prejudiciales, o por la circunstancia -probada- de que los EM -en cualquier proceso de integraciónevitan demandarse recíprocamente, o aún por la inexistencia de un órgano comunitario, es claro que nuestro Protocolo de Brasilia fue redactado con el firme propósito de evitar la intromisión de los juristas y con ello la jurisdización del Derecho Mercosur; jlamentablemente, un grave error!

\section{BIBLIOGRAFÍA}

BAPTISTA, Luiz Olavo, (árbitro del IIª laudo del TAHM), "Solução de divergências no Mercosul", en Mercosul. Seus efeitos jurídicos, económicos e políticos nos Estados membros (Maristela Basso, organizadora), 2ed., ed. Librería dos Advogados, Porto Alegre -RS-, 1997, pgs. 178-186 (157/186); FÉRNADEZ REYES, Jorge E. (ex Director de la SAM), "Evaluación de los mecanismos de solución de controversias en el Mercosur", RDM 2000-4, pgs. 159-163 (139-169).

GONÇALVES, José Botafogo, "Os Tribunais do Mercosuf", Boletim de Integração Latino-Americana N $\mathrm{N}^{\mathrm{a}}$ 18, janeiro-junho/1996, ed. MRE-GIEGETEC, Brasilia, pg. 6-7.

Mercosur/CMC/Dec. № 17/98 "Reglamento del Protocolo de Brasilia para la Solución de Controversias" (XV CMC, Río de Janeiro, 10/XII/98). Mercosur/CMC/Dec. № 25/00 "Perfeccionamiento del Sistema de Solución de Controversias del Protocolo de Brasilia". Su texto puede verse en $\mathrm{BO} 25 / \mathrm{NII} / 2000$, pgs. 30ss.

Mercosur/CMC/Dec. Na 65/00 "Perfeccionamiento del Sistema de Solución de Controversias del Protocolo de Brasilia". Su texto puede verse en BO $1 / 1 / 2001$, pgs. 11ss.

Mercosur/CMC/Dec. Na 28/94 "Tribunais Ad Hoc do Protocolo de Brasilia"; art. 1 "Os Tribunats Arbitrais Ad Hoc, a que se refere o artigo $8^{2}$ do Protocolo de Brasília, terão como sede a cidade de Assunção". 
SOARES, Guido Silva. "No es momento para una mayor institucionalización del bloque", entrevista realizada por GABRIEIA WURCEI, RDM 2000-3, pg. 36 (35-36). TAHM, asunto 1/99, Comunicados N $N^{a} 37 / 1997$ y $N^{a}$ 7/1998 del Departamento de Operaciones de Comercio Exterior (DECEX) de la Secretaria de Comercio Exterior (SECEX): Aplicación de Medidas Restrictivas al comercio recíproco, 28/IV/99. Publicado en RDM 1999-4, pgs. 257-278.

TAHM, asunto 2/99, Subsidios a la producción y exportación de carne de cerdo, 27/IX/99. Publicado en LL del 10/XII/99, pg. 2-3.

TAHM, asunto 1/00, Aplicación de Medidas de Salvaguardia sobre productos Textiles (Res. 861/99) del Ministerio de Economía y Obras y Servicios Públi$\cos (M E O y S P), 10 / \mathrm{LI} / 00$. Publicado en RDM 2000-3, pgs. 138-164. 